\title{
An asteroseismic study of the $\beta$ Cephei star $\beta$ Canis Majoris
}

\author{
M. Desmet ${ }^{1}$, M. Briquet ${ }^{1}$, A. Mazumdar ${ }^{1,2}$, C. Aerts ${ }^{1,3}$ \\ ${ }^{1}$ Instituut voor Sterrenkunde, Katholieke Universiteit Leuven, Celestijnenlaan 200 B, B-3001 Leuven, \\ Belgium \\ 2 Astronomy Department, Yale University, P.O. Box 208101, New Haven, CT 06520-8101, USA \\ ${ }^{3}$ Department of Astrophysics, University of Nijmegen, PO Box 9010, 6500 GL Nijmegen, the Netherlands
}

\begin{abstract}
We present a spectroscopic study of the $\beta$ Cephei star $\beta$ Canis Majoris. Hundreds of highresolution spectra were gathered during 4.5 years and the Si III line profiles centered on $4560 \AA$ were subjected to a detailed line profile analysis. We searched for oscillation frequencies in different line diagnostics, compared them with those known from photometric studies and concluded the presence of three frequencies in our dataset: $f_{1}=3.97933 \mathrm{~cd}^{-1}, f_{2}=$ $3.99959 \mathrm{~cd}^{-1}$ and $f_{3}=4.1832 \mathrm{~cd}^{-1}$, which were already found earlier from photometric data. By means of the moment method and from amplitude and phase variations across the profiles we could identify two of the modes as $\left(\ell_{1}, m_{1}\right)=(2,2)$ and $\left(\ell_{2}, m_{2}\right)=(0,0)$. We present our first results of modelling based on the oscillation frequencies and place constraints on the mass, age and core overshooting of this massive evolved $\beta$ Cephei star.
\end{abstract}

\section{Introduction and observations}

As already explained in Saesen et al. (2006), our work fits in with the research of asteroseismology of B-type stars. In particular, the study of $\beta$ Cephei stars is extremely useful to derive knowledge about the interior and evolution of massive stars. With this work we add $\beta$ Canis Majoris to the sample of $\beta$ Cephei stars with thorough asteroseismic modelling.

From previous studies $\beta$ Canis Majoris is known to exhibit multiperiodicity with rather low frequencies in comparison with the frequencies of other $\beta$ Cephei stars. This would indicate that $\beta$ Canis Majoris is a reasonably evolved star which makes it a particularly interesting object to study.

$\beta \mathrm{CMa}$ was observed during four and a half years with the CORALIE échelle spectrograph attached to the $1.2 \mathrm{~m}$ Euler Swiss Telescope (on La Silla ESO, Chile) and 452 high-resolution spectra were gathered. This is a unique dataset since all the previous studies were performed using photometric data. We used the first line of the $\mathrm{Si}$ III triplet with wavelength $\lambda=$ $4552.654 \AA$ and calculated the first three velocity moments $\langle v\rangle,\left\langle v^{2}\right\rangle$ and $\left\langle v^{3}\right\rangle$ (see Aerts et al. 1992 for their definition) to start our frequency analysis.

\section{Frequency analysis}

Shobbrook (1972) showed that $\beta$ Canis Majoris is multiperiodic with at least three frequencies. Handler (2005) confirmed these frequencies based on a photometric multisite campaign on $\beta$ CMa. 


\begin{tabular}{cccc}
\hline & Frequency $\left(\mathrm{cd}^{-1}\right)$ & Amplitude $\left(\mathrm{km} \mathrm{s}^{-1}\right)$ & Phase $(2 \pi \mathrm{rad})$ \\
\hline \hline$f_{1}$ & $3.97933 \pm 0.00001$ & $2.66 \pm 0.09$ & $0.354 \pm 0.005$ \\
$f_{2}$ & $3.99959 \pm 0.00003$ & $2.58 \pm 0.08$ & $0.583 \pm 0.002$ \\
$f_{3}$ & $4.1832 \pm 0.0001$ & $0.73 \pm 0.06$ & $0.953 \pm 0.003$ \\
\hline
\end{tabular}

Table 1: Frequencies of $\beta \mathrm{CMa}$ together with their amplitude and phase in the first moment. They are arranged in order of decreasing amplitude.

In our study we used the PDM (Stellingwerf 1978) and SCARGLE (Scargle 1982) methods together with the code PERIOD98 (Sperl 1998) to search for frequencies in the first three moments. We obtained the same three frequencies as Shobbrook (1972) and Handler (2005). The three frequencies, together with their amplitude and phase, are listed in Table 1 . These three frequencies together have a variance reduction of $88 \%$ in the first moment.

\section{Mode identification}

First of all, Handler (2005) performed a photometric mode identification by means of his dataset. We adopted his $\ell$-identification for $f_{1}$, which is $\ell_{1}=2$.

Telting \& Schrijvers (1997) and Schrijvers et al. (1997) showed that, when there is a minimum (almost zero) in the amplitude and a corresponding phase shift of $\pi$ near the center of the line profile, one can conclude to be dealing with a radial or a dipole mode. As one can see in Fig. 1, this certainly is the case for $f_{2}$ in our dataset. Handler (2005) found that $f_{2}$ must be a radial mode. This makes it clear that $f_{2}$ is indeed a radial mode.
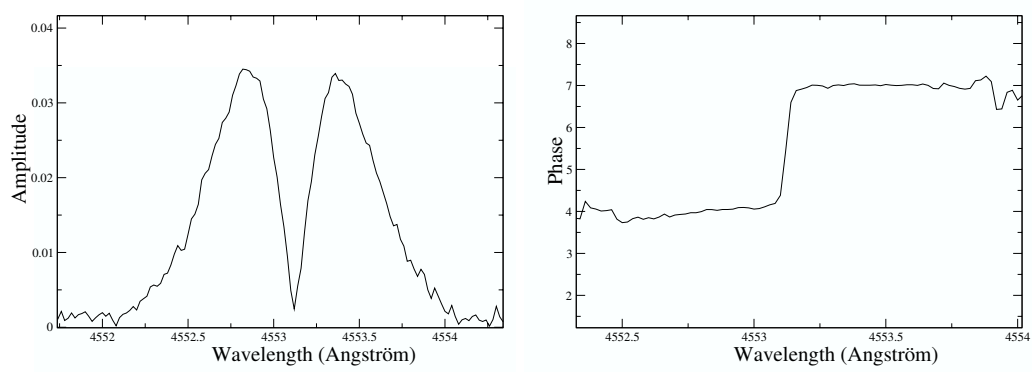

Figure 1: Amplitude and phase distribution for $f_{2}=3.99959 \mathrm{c} \mathrm{d}^{-1}$. The amplitude is unitless and given in terms of the central depth of the average line profile over the time series. The phase is expressed in $\pi$ radians.

Secondly, in order to determine the $m$-value of $f_{1}$ and the $\ell$ - and $m$-value of $f_{3}$, we continued our research with the moment method (Briquet \& Aerts 2003). With this method, the wavenumbers $(\ell, m)$ and some other continuous velocity paramaters are computed in such a way that the theoretically computed first three moment variations best fit the observed ones. We performed a multiperiodic mode identification with $f_{1}, f_{2}$, and $f_{3}$, the $\ell$-value of $f_{1}$ being fixed to $\ell_{1}=2$ and the mode of $f_{2}$ being fixed to $\left(\ell_{2}, m_{2}\right)=(0,0)$. The outcome of the moment method gives a preference to $\left(\ell_{1}, m_{1}\right)=(2,2)$, but we cannot rule out $m_{1}=1$.

In order to discriminate between $m_{1}=2$ or $m_{1}=1$, we continued as follows. By using the codes BRUCE and KYLIE (Townsend 1997) we constructed theoretical line profile time series according to the nine best moment solutions (given as a function of the wavenumbers $\ell$ 
and $m$ and the other continuous parameters). Subsequently, we computed the amplitude and phase variations across the line profile for each generated line profile time series. We then compared these theoretically computed amplitude and phase distributions with the observed ones for $f_{1}$. From this comparison, we concluded that the wavenumbers of the main mode are clearly $\left(\ell_{1}, m_{1}\right)=(2,2)$. Unfortunately we could not conclude anything concerning the mode with frequency $f_{3}$.

The moment method gives also indirect information about the equatorial rotation velocity. By closely examining all the given values, we could also make an estimation of the equatorial rotation velocity: $v_{e q}=32.5 \pm 2.5 \mathrm{~km} \mathrm{~s}^{-1}$.

\section{Modelling of the frequencies}

Our goal is to construct stellar models which show oscillations in accordance with the observed modes of $\beta$ Canis Majoris. By doing that, we hope to derive constraints on some stellar parameter values such as mass, age and the convective overshoot parameter.

We calculated an extensive grid of standard non-rotating stellar models using the evolutionary code CESAM (Morel 1997). We used the opacities derived from the OPAL code (Iglesias \& Rogers 1996). We adopted the OPAL equation of state (Rogers \& Nayfonov 2002). Convection was described by the standard mixing length theory (Henyey et al. 1965) and nuclear reaction rates were obtained from the NACRE compilation (Angulo et al. 1999). The $\mathrm{p}$ - and g-mode oscillation frequencies of low degree modes $(\ell=0,1,2,3)$ were computed for each model under the adiabatic approximation, using the pulsation package, ADIPLS (Christensen-Dalsgaard \& Berthomieu 1991). Models in the grid are parameterised by mass, age, the initial chemical composition $\left(X_{0}, Z_{0}\right)$ and the convective overshoot parameter $\left(\alpha_{\mathrm{ov}}\right.$, measured in terms of local pressure scale height).

Since we do not know the mode corresponding to the third frequency $f_{3}$, we can only try to fit the two frequencies $f_{1}$ and $f_{2}$ at this moment. Besides matching these two frequencies, we took into account an additional observational constraint: the position in the HertzsprungRussel (HR) diagram. From an extensive search in the literature, we derived a range for the effective temperature of $\log T_{\text {eff }}=4.40 \pm 0.02$ (Heynderickx et al. 1994, De Cat 2002) and from a given parallax $(\pi=6.53 \pm 0.66$ mas, Perryman \& ESA 1997) and visual magnitude $\left(\mathrm{m}_{v}=1.97 \mathrm{mag}\right.$, Johnson et al. 1966), we calculated a range for the luminosity of $\log L / L \odot \in$ $[4.25,4.57]$. We also obtained a value for the metallicity: $[\mathrm{m} / \mathrm{H}]=0.04 \pm 0.10$ (DaszyńskaDaszkiewicz \& Niemczura 2005; Niemczura \& Daszyńska-Daszkiewicz 2005). We considered two values for $\alpha_{\mathrm{ov}}: 0.0$ and 0.2 and three values for the metallicity $Z:-0.06,0$ and 0.14 . We calculated evolutionary tracks with masses between 12 and $15 M_{\odot}$.

First of all, we fitted the radial mode corresponding to $f_{2}$. For each model in the correct position in the HR diagram, we compared the given frequencies corresponding to $\ell=0$ but with different radial order $n$ to $f_{2}$. We concluded that $f_{2}$ must be the radial fundamental frequency, since higher radial orders are not compatible with the star's position in the HR diagram.

Subsequently, we compared $f_{1}$ with the frequencies corresponding to $\ell=2$ in the models which reproduce the correct fundamental radial frequency. Since non-rotating stellar models only reproduce frequencies corresponding to $m=0$ and $m_{1}=2$, we took the effect of rotation into account, by calculating the splitting value $\delta \nu$, which is a function of $m_{1}, v_{e q}$, the radius $R$ and the Ledoux constant in the first order approximation. By means of the constraint of the position of $\beta \mathrm{CMa}$ in the HR diagram, we obtained an estimate for the radius: $R=9.0 \pm 0.35 R_{\odot}$. The Ledoux constant is estimated from the eigenfunction of $\ell=2$ modes. After research of a number of representative models, we reached the conclusion that the radial order of $f_{1}$ equals $n_{1}=-1$ and comes from the first g-mode. With all this information, we could calculate the actual splitting value $\delta \nu$ and could start fitting the two frequencies together. 
The first results of our modelling are the following. We point out that they have to be considered as provisional results. We were able to construct some stellar models for which the two relevant frequencies match the observed ones. An important outcome, however, is that the convective overshooting of the core is substantial $\left(\alpha_{\mathrm{ov}} \gtrsim 0.2\right)$. The mass of $\beta$ Canis Majoris probably lies between 13.8 and $14.5 \mathrm{M}_{\odot}$ and the age of $\beta \mathrm{CMa}$ approximately lies between $10 \times 10^{6}$ and $11.5 \times 10^{6}$ years.

\section{Conclusion and future work}

We performed a detailed asteroseismic study of $\beta$ Canis Majoris. The frequency analysis led to the extraction of three frequencies. We could well identify the modes of the two dominant frequencies. We constructed stellar models which reproduce frequencies matching our observed frequencies. Thus, we could deduce a number of constraints concerning the mass, radius, age and convective overshoot parameter of $\beta$ Canis Majoris.

These constraints are being checked more thoroughly in ongoing work. We also hope to consider the question of the excitation of the observed modes in our models in a future study.

Acknowledgments. This work was based on observations obtained with the CORALIE échelle spectrograph attached to the $1.2 \mathrm{~m}$ Euler telescope (La Silla, Chile). MB is a Postdoctoral Fellow of the Fund for Scientific Research, Flanders. MD, AM and CA are supported by the Research Fund, K. U. Leuven under the grant GOA/2003/04.

\section{References}

Aerts, C., De Pauw, M., Waelkens, C. 1992, A\&A 266, 294

Angulo, C., Arnould, M. \& Rayet, M. (NACRE collaboration) 1999, Nuclear Physics A 656, 1

Briquet, M. \& Aerts, C. 2003, A\&A 398, 687

Christensen-Dalsgaard, J. \& Berthomieu, G. 1991, Solar interior and atmosphere, University of Arizona Press, 401

Daszyńska-Daszkiewicz, J. \& Niemczura, E. 2005, A\&A 433, 1031

De Cat, P. 2002, in "Radial and Nonradial Pulsations as Probes of Stellar Physics", eds. C. Aerts, T.R. Bedding, J. Christensen-Daldgaard, ASP Conf. Ser. 259, 197

Handler, G. 2005, Private communication

Henyey, L., Vardya, M. S. \& Bodenheimer, P. 1965, ApJ 142, 841

Heynderickx, D., Waelkens, C., \& Smeyers, P. 1994, A\&A 105, 447

Iglesias, C. A., Rogers, F. J. 1996, ApJ 464, 943

Johnson, H. L., Iriarte, B., Mitchell, R. I., \& Wisniewskj, W. Z. 1966, Communications of the Lunar and Planetary Laboratory 4, 99

Morel, P. 1997, A\&AS 256, L5

Niemczura, J. \& Daszyńska-Daszkiewicz, E. 2005, A\&A 433, 659

Perryman, M. A. C. \& ESA. 1997, Noordwijk, Netherlands: ESA Publications Division, 1997, Series: ESA SP Series vol no: 1200

Rogers, F. J., \& Nayfonov, A. 2002, ApJ 576, 1064

Saesen, S., Briquet, M., Aerts, C. 2006, This volume

Scargle, J. D. 1982, AJ, 263835

Schrijvers, C., Telting, J. H., Aerts, C., Ruymaekers, E., \& Henrichs, H. F. 1997, A\&AS 121, 343

Shobbrook, R. R. 1972, MNRAS 161, 257

Sperl, M. 1998, CoAst 111, 1

Stellingwerf, R. F. 1978, AJ 224, 953

Telting, H. H. \& Schrijvers, C. 1997, A\&A 317, 723

Townsend, R. H. D. 1997 MNRAS 284, 839 